\title{
ACTIVE FLOW CONTROL ON A BOUNDARY-LAYER-INGESTING INLET
}

\author{
Susan Althoff Gorton*, Lewis R. Owens*, Luther N. Jenkins ${ }^{\dagger}$, Brian G. Allan* \\ NASA Langley Research Center \\ Hampton, Virginia \\ Ernest P. Schuster \\ The Boeing Company \\ Huntington Beach, California
}

\begin{abstract}
Boundary layer ingestion (BLI) is explored as a means to improve overall system performance for a Blended Wing Body configuration. The benefits of BLI for vehicle system performance benefit are assessed with a process derived from first principles suitable for highly-integrated propulsion systems. This performance evaluation process provides a framework within which to assess the benefits of an integrated BLI inlet and lays the groundwork for higher-fidelity systems studies. The results of the system study show that BLI provides a significant improvement in vehicle performance if the inlet distortion can be controlled, thus encouraging the pursuit of active flow control (AFC) as a BLIenabling technology.
\end{abstract}

The effectiveness of active flow control in reducing engine inlet distortion was assessed using a $6 \%$ scale model of a $30 \%$ BLI offset, diffusing inlet. The experiment was conducted in the NASA Langley Basic Aerodynamics Research Tunnel with a model inlet designed specifically for this type of testing. High mass flow pulsing actuators provided the active flow control. Measurements were made of the onset boundary layer, the duct surface static pressures, and the mass flow through the duct and the actuators. The distortion was determined by 120 total pressure measurements located at the aerodynamic interface plane. The test matrix was limited to a maximum freestream Mach number of 0.15 with scaled mass flows through the inlet for that condition. The data show that the pulsed actuation can reduce distortion from $29 \%$ to $4.6 \%$ as measured by the circumferential distortion descriptor DC60 using less than $1 \%$ of inlet mass flow. Closed loop control of the actuation was also demonstrated using a sidewall surface static pressure as the response sensor.

\footnotetext{
* Member AIAA, Research Engineer, Flow Physics and Control Branch

${ }^{\dagger}$ Research Engineer, Flow Physics and Control Branch

${ }^{\ddagger}$ Chief Engineer, Propulsion Concepts
}

\section{INTRODUCTION}

The effect of aviation on the environment and in particular global warming has recently become a focus of study ${ }^{1}$. In response to environmental concerns and to foster revolutionary propulsion technologies, NASA launched the Ultra Efficient Engine Technology (UEET) program in late $1999^{2}$. This program has several elements, one of which is to explore the feasibility of the Blended-Wing-Body (BWB) concept as an efficient alternative to conventional transport configurations. The BWB concept has been considered in various forms for several years. ${ }^{3-6}$ Studies have shown that in order to make the largest impact on the vehicle performance the engines and inlets should be placed near the upper surface on the aft section of the vehicle. However, the incorporation of the inlets on the surface of the vehicle increases the technical risk of the configuration ${ }^{5}$. Although Boeing reduced this risk by positioning the engines on pods for most of their configuration studies, the NASA UEET program has continued to pursue the goal of boundary-layeringesting (BLI) offset inlets for the BWB. Additional system studies, sponsored by NASA, continue to indicate the additional advantages of BLI inlet for the configuration including less fuel burn and lower noise characteristics. The configuration of the BWB with BLI is shown in Refs. 5 and 7 and pictured in Figure 1.

When the engines are positioned near the surface, the BWB engine inlet will likely be an S-duct inlet with the capability to ingest the large boundary layer that will build up over the aircraft body. The inlet must perform this task in a manner compatible with engine performance requirements for distortion and pressure recovery. Since the boundary layer on the BWB is expected to be on the order of $30 \%$ of the inlet height, this presents a challenging task for inlet design. In addition, the performance assessment of such a highly-integrated propulsion system is a complex undertaking, requiring the simultaneous 
examination of many influential factors in order to determine whether BLI provides a benefit from a system standpoint. The trade-offs among reduced drag, weight savings or penalty, and engine operation must all be considered to assess the relative benefit of BLI technology. However, the engine must be able to operate acceptably in the BLI environment. Therefore, an acceptable level of distortion for engine operation must be achieved even if the engine operates at reduced efficiency levels.

This requirement for at least a minimum level of inlet performance under the severe conditions of an adverse pressure gradient from the S-duct and a very large onset boundary layer flow have led to the consideration of flow control devices in the inlet to control the flow in this type of configuration. Passive flow control in the form of micro vortex generators or microvanes can be used to improve the inlet flow. ${ }^{7-12}$ Reference 7 discusses work using passive devices for an S-duct with BLI while active flow control methods have also been investigated as a means to improve inlet flow for aggressive serpentine inlets with minimal BLI. ${ }^{11-12}$

Much research ${ }^{13-25}$ is already underway to identify and develop active flow control devices and technologies for a variety of applications. This recent emphasis on active flow control and the successful progress in development of actuators, design tools, and control methodologies encouraged the hypothesis that a significant inlet boundary layer could be managed and improved by the application of active flow control.

The purpose of the present investigation was to demonstrate through a first principles analysis that BLI inlets with a nominal distortion penalty would be expected to improve the vehicle performance of a BWB configuration. With this performance improvement in mind, the next step in the research program was to demonstrate for a $30 \%$ BLI offset inlet that active flow control can provide a nominal distortion level to the engine. Thus, active flow control becomes an enabling technology for the application and benefit of BLI inlets.

The process to assess the benefits of BLI was developed from first principles using a control volume approach to capture the integratedthrust/drag-force accounting. The Breguet range equation $^{26}$ is used to assess the impact of BLI inlet technology by considering various factors that contribute to the system performance. This initial assessment of BLI benefits for a BWB system can be further refined as better data become available.

The experimental active flow control study was conducted at low Mach numbers in order to demonstrate the concept before progressing to representative flight Mach numbers. During the experiment, an inlet with $30 \%$ BLI was tested at a free stream Mach number of 0.15 for several inlet mass flows. Pulsed, high mass flow actuators and micro vortex generators were used to provide active and passive flow control. Measurements of inlet distortion and pressure recovery were made at the Aerodynamic Interface Plane (AIP).

\section{BENEFIT OF BLI}

The effect of BLI on engine performance is known to be detrimental because BLI increases the distortion and reduces the pressure recovery at the inlet exit. ${ }^{27}$ However, the overall system benefits of BLI must be analyzed to determine whether there is a benefit from BLI for a configuration. Reference 5 cites work done early in the development of the BWB configuration that indicated BLI might improve performance as much as $10 \%$. As NASA has continued to explore the benefits of BLI for the BWB, there have been continued efforts to assess the BLI benefits in a progressively higher fidelity manner. As part of the systems benefits assessment, NASA sponsored a contract with The Boeing Company in 2001 to assess the BLI benefits for a representative BWB configuration. The method and findings presented here were the result of that contract.

Control volumes are established for both the baseline and the BLI inlet concepts. The fundamental governing equations of conservation of mass, momentum, and energy and the force balance equation are the basis for the analysis. An engine flow thermodynamic analysis is performed on a two dimensional representation of the combined vehicle and propulsion system configuration. The momentum deficit produced by the boundary layer is included in the control volume analysis for the BLI configuration. As well, the installed engine performance due to BLI is accounted for in the specific fuel consumption (SFC) and net thrust levels for the engine performance. The baseline was a pylon-mounted engine configuration. The control volume surfaces are shown in Figure 2 
The combined effect of the momentum deficit along with the associated configuration effects is analyzed using the Breguet range equation over relatively short range cruise segments to simplify the comparative analysis between the baseline and BLI installations. Baseline conditions representative of a 450 passenger vehicle were assumed for lift/drag, initial weight, final weight, velocity and range. Fuel burn weight decrement was selected to be constant to observe the impact of BLI integration on range. Propulsion system integration factors were incremented between the baseline and BLI configurations to track the changes due to BLI. Table 1 shows the summary of the propulsion system integration factors included in this analysis. The fidelity of the entire analysis is determined to a great extent by the fidelity of the analysis for each integration component. For this study, although many components were included to represent integration effects, there were many initial simplifying assumptions made for the factors because of the complexity of determining the magnitude of their effects. As more information becomes available, the uncertainty range of the results in the analysis is reduced.

The method assumes that the application of active flow control will decrease distortion of the inlet to a level that allows acceptable engine operation. Therefore, the method does not assess distortion as one of the factors. This is the underlying assumption that is included in most systems analysis of BLI that presumes that a technology such as active flow control will enable operations in a BLI environment. Thus the systems analysis only assesses the effect of $\mathrm{BLI}$; the AFC performance is presumed in the basic analysis.

For each term in the Breguet equation, a delta due to the application of BLI was determined and the change in range was computed. The percent improvement in range for $30 \%$ BLI for several future engine design by-pass ratios is shown in Figure 3. With current technology levels near a bypass ratio of 10 , this analysis shows an expected benefit of nearly $13 \%$ for the cruise portion of the profile. This benefit is significant enough to justify the pursuit of the active flow control technology to enable BLI for the configuration.

\section{EXPERIMENTAL APPARATUS AND METHODS}

Facility and Model

The experiment was conducted in two phases in the NASA Langley Basic Aerodynamics Research Tunnel (BART). This tunnel is an open return, atmospheric facility used primarily for fundamental flow physics research. The maximum flow speed entering the test section is approximately Mach = 0.17 .

The model was located at a station 67 inches downstream of the start of the test section so that the natural boundary layer buildup of the facility could be used to simulate the boundary layer ingestion height of $30 \%$ of the inlet height. The tunnel boundary layer was allowed to transition naturally; measurements during previous testing have indicated the boundary layer is fully turbulent at station 50 . The boundary layer height was verified by using a boundary layer rake to measure the onset boundary layer to the inlet during the test program. The boundary layer profile directly in front of the inlet is shown in Figure 4. Analysis of the profile using Coles wake parameter ${ }^{28}$ indicates the flow is experiencing an adverse pressure gradient and the thickness of the boundary layer is 1.5 inches or $36 \%$ of the inlet height. Boundary layer data obtained to the side of the inlet indicated the same height but the wake parameter indicated a zero pressure gradient.

The inlet model design and features are shown in Figures 5-6. Figure 5 illustrates how the model inlet was mounted on the ceiling of the tunnel and breached the tunnel ceiling. The inlet installed in the tunnel is shown in Figure 6. The inlet piece was constructed using stereo lithography and had no seams. The inlet was covered with a fiberglass cowl in the tunnel, and the pressure boundary between the model and the tunnel wall was sealed. A centrifugal blower provided the source for drawing air through the inlet and a calibrated venturi system measured the mass flow.

The inlet characteristics are listed in Table 2 and shown in Figure 7. The inlet was designed in 2000 by Boeing under contract to NASA to provide an inlet that was representative of a general class of inlets that could be considered for application in a commercial BWB configuration. This inlet design is generic in nature and is an open geometry that can be used for computational validation and experimental studies. This inlet geometry has also been tested at 
high Mach number and Reynolds number during a different phase of the UEET program. ${ }^{29}$

The inlet was instrumented with surface pressure ports along the top, bottom and sidewalls of the duct. A 40 probe total pressure rake shown in Figure $6 \mathrm{~b}$ was positioned at the AIP to measure pressure recovery and distortion. The total pressure rake was designed to conform to the Society of Automotive Engineers (SAE) standard $^{30}$; in addition, the rake could be clocked at 15 and 30 degrees to provide a higher resolution measurement. The rake arms were airfoil shaped and had less than $10 \%$ blockage in the inlet. The probes on the rake and along the wall were connected to an electronic scanning pressure measurement system.

\section{Flow Control Devices}

Based on a series of risk reduction tests, ${ }^{25}$ two flow control devices were considered for their effectiveness in controlling the flow characteristics of the inlet. Microvanes have been shown by experiment and computational effort to be effective in controlling secondary flow and improving pressure recovery and distortion ${ }^{7-12}$. However, there are indications that active flow control could provide better flow control characteristics, tailoring of flow management to flight condition, and eliminate concerns such as foreign object damage, icing possibilities and maintenance issues that hamper the application of micro vortex generators ${ }^{12-13}$.

In order to provide a baseline passive device for flow control comparisons, micro vortex generators (MVG's) were installed inside the inlet. The MVG's were approximately $13 \%$ of the onset boundary layer height and were arranged in a co-rotating pattern at an angle of 22 degrees to the onset flow with the inlet lower surface centerline providing a plane of symmetry. The MVG's were located at a distance of 0.25 inches on either side of the lower surface centerline with a 0.625 inch spacing between each, following spacing guidelines ${ }^{9}$ and were located at the same longitudinal station as the active flow control jets. With this layout, ten MVGs were present on the lower surface.

The active flow control actuators were a high-massflow, pulsing actuator that consisted of basically an electronically-controlled needle valve. The actuators could be controlled in frequency from $20 \mathrm{~Hz}$ to 200 $\mathrm{Hz}$ and the duty cycle could be varied from $20 \%$ to $80 \%$. The actuators required a pressure differential to operate, and the pressure differential, duty cycle, and frequency affected the amount of mass flow that could be passed through each actuator. For this test program, eight actuators were operated from a common manifold as shown in Figure 8. For the cases presented here, the pressure into the actuator manifold was 100 psig.

The output of each actuator was directed through different combinations of connectors, tubing, and tees to the ports that were located in the model. The 32 port locations are shown in Figure 9. Each port was installed at an angle of $30 \mathrm{deg}$ to the surface tangent at $90 \mathrm{deg}$ to the onset flow. There were two longitudinal locations of the actuator ports. The upstream location was at $\mathrm{X} / \mathrm{L}$ of 0.36 and the downstream location was at $\mathrm{X} / \mathrm{L}$ of 0.54 . Various combination of actuator ports were evaluated during the test program, and a Design of Experiment (DOE) matrix was used in addition to the traditional one factor at a time method in order to establish the optimum performance for these jets in this inlet with these port location options. The ports for the bestperforming configuration, which were located at the upstream station only, are indicated in Figure 9. When a port was not being used, the port was sealed. The total mass flow of the actuators for any given configuration was measured using a flow meter upstream of the manifold. A settling tank was used during the initial testing to ensure that unsteady flow oscillations did not feedback and influence the mass flow reading upstream. Once it was established that the flow meter was unaffected by the pulsed actuators, the settling tank was removed from the system in order to improve the system response to changes in commanded mass flow.

The actuators were computer controlled through a function generator and a switchbox. The actuators could be individually activated; however, all the active actuators were operated at the same frequency and duty cycle. Sweeps of duty cycle, frequency, and mass flow were made to determine the best operating conditions. A square wave signal was the control signal to the actuators.

A dynamic pressure transducer was installed near the actuator port for one of the actuators to measure the pressure signal that was injected into the flow. Although the input signal was a square wave, the pressure signal was attenuated through the tubing, and the signal that was injected into the flow had more of a shark fin shape as shown in Figure 10. 
Data Acquisition

The tunnel parameters were acquired using standard wind tunnel measurement techniques and the accuracy of the measurements is reported in Table 3. The accuracy was assessed using the method of Reference 31.

\section{Test Conditions}

The main test conditions were established by setting the tunnel Mach number and the mass flow through the inlet. Tufts were used to verify the minimum mass flow rate that resulted in a smooth flow entering the inlet. Two primary free stream Mach numbers were tested, 0.10 and 0.15 . Mass flow in the inlet was varied from 1250 to 1800 standard cubic feet per minute (SCFM), corresponding to the inlet mass flow requirement for the throat Mach number. The inlet throat Mach numbers were close to the respective free stream Mach numbers. The actuators were operated through ranges of frequency, mass flow, and duty cycle to determine the optimum performance for the given jet orifice layout.

\section{DISCUSSION OF RESULTS}

Data were obtained for several different test conditions during this investigation. In this paper, the baseline flow distortion and pressure recovery are compared with the active flow control configurations for the highest Mach number of 0.15 and the highest inlet mass flow, 1800 SCFM, because these conditions represent a realistic inlet mass flow ratio operating condition. The inlet mass flow of 1800 SCFM was scaled for the inlet throat Mach number of 0.15. Data are presented for several different actuator mass flow settings.

\section{Baseline Flow Field}

Figure 11 shows the contours of total pressure ratios for the baseline with $\mathrm{M}=0.15$ and $1800 \mathrm{SCFM}$ inlet mass flow. The distortion as calculated using all three rake clock positions with 120 pressure measurements and the DC60 method m $^{27}$ is $29 \%$. The distortion calculated using the Aerospace Recommended Practice (ARP) 1420 standard circumferential distortion intensity element DPCP with the 40 probe rake positions is 0.0025 . Pressure recovery for this case is 0.997 . The effect of the onset boundary layer can be seen in the lower total pressure ratio levels at the bottom of the inlet. Because the duct geometry is relatively mild and the Mach numbers in this case are low, no separation was identified from the inlet wall surface pressures.

\section{Passive Flow Control Devices}

MVGs were placed at the locations described above and the resulting distortion is shown in Figure 12 for a Mach number of 0.15 and an inlet flow of 1800 SCFM. For this condition, the MVGs reduce the distortion to a value of $10.8 \%$ DC60 and 0.0014 DPCP. There was no attempt during this program to optimize the performance of the MVGs. Rather, they were evaluated to provide a benchmarking distortion goal for the active flow control devices.

\section{Active Flow Control Devices}

Figure 13 presents the contours of total pressure ratios for the active flow control case with $\mathrm{M}=0.15$, inlet mass flow $=1800$ SCFM, and the actuators operating at $0.6 \%$ inlet mass flow, $50 \mathrm{~Hz}$, and $80 \%$ duty cycle. The DC60 value of distortion is $4.6 \%$. The ARP 1420 distortion (DPCP) is 0.000 where round-off error causes the calculation of DPCP to be difficult using standard tools. Pressure recovery for this case is still 0.997. Although the pressure recovery is unchanged due to the application of active flow control, the distortion is significantly improved. This improvement demonstrates the ability of active flow control to enable the application of BLI technology to a configuration with minimal distortion penalty.

There were two assumptions that constrained the experimental portion of this program. The first was that active flow control could only use up to $1 \%$ of the inlet mass flow. This constraint was selected to minimize the impact of an active flow control system on the overall vehicle. The second assumption, obtained from industry, was that the maximum distortion level for engine operation was 10\% DC60. The information indicated that a distortion level below the $10 \%$ DC60 was essential, but that distortion lower than $10 \%$ did not increase the benefit.

With these assumptions in mind, controlling the distortion in the inlet was examined from two different perspectives: the first was to determine the minimum distortion that can be achieved with actuator mass flow at $1 \%$ of inlet mass flow; the second was to identify the minimum mass flow that was required to achieve a distortion level below $10 \%$. Figure 14 shows an actuator mass flow sweep and the resulting distortion for the best actuator configuration. The actuator mass flow is the total mass flow through all the actuators. There is a 
minimum distortion level at about $0.55 \%$ of inlet mass flow; above this level, the distortion increases for increasing actuator mass flow. The minimum mass flow to achieve a distortion level below 10\% was $0.4 \%$. These levels of actuator mass flow required and inlet distortion achieved indicate that active flow control may be a viable option for controlling distortion and enabling BLI as an option for various configurations. As discussed above, if the engine distortion can be controlled with AFC, the systems analysis study shows a significant benefit for the BLI configuration.

One of the benefits of active flow control over passive flow control methods is the ability to tailor the amount of active flow control applied for a given flow condition. The application of this would in most cases require some type of control system. For this research effort, a closed loop control system was developed to demonstrate the ability of the actuators to establish and hold a commanded distortion. The control system diagram is shown in Figure 15. A series of sensitivity sweeps were run to obtain the system parameters such as gain and linearity. For the initial control system, an on-line $\mathrm{DC} 45^{27}$ calculated from the 40-probe rake was used as the control feedback sensor. The commanded distortion and the actual distortion tracked very closely for this configuration, as shown in Figure 16a.

For the final control system, a surface pressure near the AIP was used as the control feedback sensor. This surface pressure had shown a linear relationship to DC45 in the sensitivity sweeps. Using the surface pressure as the sensor, the commanded distortion and the actual distortion still tracked together fairly well, as shown in Figure 16b. This indicates that the active flow control system could be closed-loop controlled using only a pressure sensor in the wall of the inlet without any measurement disturbance in the inlet flow.

\section{CONCLUSIONS}

The purpose of the present investigation was to assess the potential performance benefit of $30 \%$ boundary layer ingestion (BLI) when applied to a Blended Wing Body (BWB) configuration and to demonstrate that active flow control can be effective as an enabling technology for BLI. The benefits of BLI were assessed with a method developed from first principles using a control volume approach to capture the force accounting and using the Breguet range equation to assess the impact of BLI inlet technology. Active flow control was demonstrated using an inlet with $36 \%$ BLI tested at a free stream Mach number of 0.15 with an inlet mass flow scaled for approximately the same throat Mach number. Pulsed, high-mass-flow actuators and micro vortex generators were used to provide active and passive flow control. The following conclusions are made:

1. The benefit of BLI to a representative, 450passenger vehicle was an improvement in cruise range on the order of $13 \%$ for a bypass ratio 10 engine with $30 \%$ BLI. This is a significant enough improvement to encourage the pursuit of the active flow control enabling technology.

2. For $\mathrm{M}=0.15$, the application of active flow control pulsed jets operating at $0.55 \%$ inlet mass flow in the inlet reduced the distortion from a DC60 value of $29 \%$ for the baseline to $4.6 \%$ for the active flow control case.

3. The effectiveness of the active flow control pulsed jets had a maximum at an actuator mass flow rate of $0.55 \%$ of the inlet mass flow.

4. The minimum actuator mass flow rate that resulted in a DC60 value of $10 \%$ or less was $0.4 \%$ of the inlet mass flow.

5. A closed-loop controller was demonstrated that used a surface static pressure as the feedback control sensor.

\section{ACKNOWLEDGEMENTS}

This research was supported by the NASA Ultra Efficient Engine Technology (UEET) Highly Integrated Inlet (HII) project. Special thanks to Mr. Richard D. White, Jr. for his contributions to the experimental effort.

\section{REFERENCES}

1. Johnson, T., “Aviation's Environmental Impact on the Global Atmosphere," Proceedings of Aviation and the Environment - Their Future in an Integrated Transport Policy, RAE, London, 1999, pp. 13.1-13.5. 2. Brown, A. S., "HSR Work Propels UEET Program (High Speed Research in Ultraefficient Engine Technology in Aircraft Industry)," Aerospace America, Vol. 37, No. 5, May 1999, pp. 48-50.

3. Callaghan, J. T., and Liebeck, R. H., "Some Thoughts on the Design of Subsonic Transport Aircraft for the $21^{\text {st }}$ Century," Cockpit, Dec. 1990, pp. 5-13.

4. Liebeck, R. H., Page, M. A., Rawdon, B. K., "Evolution of the Revolutionary Blended-WingBody," Transportation Beyond 2000: Technologies 
Needed for Engineering Design," February, 1996, pp. 431-459.

5. Liebeck, R. H.,'Design of the Blended-WingBody Subsonic Transport," AIAA 2002-002.

6. Daggett, D. L., "Ultra Efficient Engine Technology Systems Integration and Environmental Assessment," NASA CR-2002-211754, July 2002.

7. Anabtawi, A. J., Blackwelder, R. F., Lissaman, P. B. S., Liebeck, R. H., "An Experimental Investigation of Boundary Layer Ingestion in a Diffusing S-Duct With and Without Passive Flow Control," AIAA 99-0739.

8. Anderson, B. H., and Gibb, J., "Vortex Generator Installation Studies on Steady State and Dynamic Distortion," AIAA 96-3279, July, 1996.

9. Lin, J. C., "Review of Research on Low-Profile Vortex Generators to Control Boundary-Layer Separation," Progress in Aerospace Sciences, Vol 38, p 389-420, 2002.

10. Anderson, B. H., Baust, H. D., and Agrell, J., "Management of Total Pressure Recovery, Distortion and High Cycle Fatigue in Compact Air Vehicle Inlets," NASA TM-2002-212000, December, 2002.

11. Anderson, B. H., Miller, D. N., Yagle, P. J., and Truax, P. P., "A Study on MEMS Flow Control For the Management of Engine Face Distortion in Compact Inlet Systems," Proceedings of the $3^{\text {rd }}$ ASME/JSME Joint Fluids Engineering Conference, July, 1999.

12. Hamstra, J. W., Miller, D. N., Truax, P. P., Anderson, B. H., and Wendt, B. J., "Active Inlet Flow Control Technology Demonstration," The Aeronautical Journal of the Royal Aeronautical Society, October, 2000.

13. Lord, W. K., MacMartin, D. G., and Tillman, T. G., "Flow Control Opportunities in Gas Turbine Engines," AIAA 2000-2234.

14. Vakili, A. D., Wu, J. M., Liver, P., and Bhat, M. K., "Flow Control in a Diffusing S-Duct," AIAA 850524 .

15. Smith, B. L., and Glezer, A., "The Formation and Evolution of Synthetic Jets," Physics of Fluids, Vol. 10, No. 9, 1998.

16. Barberopoulos, A. A., and Garry, K. P., "The Effect of Skewing on the Vorticity Produced by an Airjet Vortex Generator," The Aeronautical Journal, v 102, no 1013, March 1998, p 171-179..

17. Tilmann, C. P., Langan, K. J., Betterton, J. G., and Wilson, M. J., "Characterization of Pulsed Vortex Generator Jets for Active Flow Control," Presented at the RTO AVT Symposium on Active Control Technology for Enhanced Performance
Operation Capabilities of Military Aircraft, Land Vehicles and Sea Vehicles, Germany, May, 2000.

18. Peake, D. J., Henry, F. S., and Pearcey, H. H., "Viscous Flow Control with Air-Jet Vortex Generators," AIAA 99-3175, June, 1999.

19. Crook, A., and Wood, N. J., "Measurements and Visualizations of Synthetic Jets," AIAA 2001-0145.

20. Wygnanski, I., "Some New Observations Affecting the Control of Separation by Periodic Excitation," AIAA 2000-2314, June, 2000.

21. Washburn, A. E., Gorton, S. A., and Anders, S. G., "Snapshot of Active Flow Control Research at NASA Langley," AIAA 2002-3155.

22. Schaeffler, N. W., Hepner, T. E., Jones, G. S., and Kegerise, M. A., "Overview of Active Flow Control Actuator Development at NASA Langley Research Center," AIAA 2002-3159.

23. Sellers, W. L., III, Jones, G. S., and Moore, M. D., "Flow Control Research at NASA Langley in Support of High-Lift Augmentation," AIAA 2002606.

24. Rao, N. M., Feng, J., Burdisso, R. A., and Ng, W. F., "Experimental Demonstration of Active Flow Control to Reduce Unsteady Stator-Rotor Interaction," AIAA Journal, v 39, no 3, March 2001, p 458-464.

25. Jenkins, L. N., Gorton, S. A., and Anders, S. G., "Flow Control Device Evaluation for an Internal Flow with an Adverse Pressure Gradient," AIAA 2002-0266.

26. McCormick, B. W., Aerodynamics, Aeronautics, and Flight Mechanics, John Wiley \& Sons, New York, 1979, p. 441-442.

27. Seddon, J., and Goldsmith, E. L., Intake Aerodynamics, Second Edition, AIAA, Reston, Virginia, 1999, p. 169, 278-280.

28. White, F. M., Viscous Fluid Flow, Second Edition, McGraw-Hill, Inc., New York, 1991, p. $417-$ $420,450$.

29. Berrier, B. L., "Evaluation of Flush-Mounted, SDuct Inlets with Large Amounts of Boundary Layer Ingestion," RTO Vehicle Propulsion Integration Symposium, Warsaw, Poland, October, 2003.

30. Gas Turbine Engine Inlet Flow Distortion Guidelines. Aerospace Recommended Practice 1420B, SAE International, 2001.

31. Instruments and Apparatus. "Part I Measurement Uncertainty," ANSI/ASME PTC 19.11985, American National Standards Inst., 1985. 
Table 1. Factors for Breguet Range Increment.

\begin{tabular}{|c|c|c|c|}
\hline LIFT/DRAG & $\begin{array}{c}\text { SPECIFIC FUEL } \\
\text { CONSUMPTION }\end{array}$ & INITIAL WEIGHT & FINAL WEIGHT \\
\hline $\begin{array}{c}\text { Nacelle Aftbody Pressure } \\
\text { Drag }\end{array}$ & Bypass Ratio (Cycle) & $\begin{array}{c}\text { Active Flow Control } \\
\text { System Weight }\end{array}$ & $\begin{array}{c}\text { Same Delta fuel for both } \\
\text { systems }\end{array}$ \\
\hline Nacelle Skin Friction & Pressure Recovery & Nacelle Weight & \\
\hline Pylon Skin Friction Drag & Momentum Benefit & Engine Weight & \\
\hline $\begin{array}{c}\text { Body Surface Nacelle } \\
\text { Footprint Drag }\end{array}$ & & Mount Weight & \\
\hline Drag due to Lift & & Pylon Weight & \\
\hline
\end{tabular}

Table 2. Inlet characteristics

\begin{tabular}{|l|l|}
\hline Average wall angle, deg $(\phi)$ & 19.4 \\
\hline Inlet length, in $(\mathrm{L})$ & 18.48 \\
\hline Inlet offset height, in $(\Delta \mathrm{H})$ & 6.24 \\
\hline Exit diameter, in $(\mathrm{D})$ & 6.00 \\
\hline Inlet throat area, sq in $\left(\mathrm{A}_{\mathrm{i}}\right)$ & 26.45 \\
\hline Inlet highlight area, sq in $\left(\mathrm{A}_{\mathrm{HL}}\right)$ & 34.62 \\
\hline Diffuser exit area, sq in $\left(\mathrm{A}_{2}\right)$ & 28.28 \\
\hline Inlet throat height, in $\left(\mathrm{H}_{\mathrm{i}}\right)$ & 4.17 \\
\hline Inlet throat width, in $\left(\mathrm{W}_{\mathrm{i}}\right)$ & 7.97 \\
\hline Inlet lip length, in $(\mathrm{a})$ & 1.18 \\
\hline Inlet lip height, in $(\mathrm{b})$ & 0.59 \\
\hline Cowl forebody length, in $(\mathrm{X})$ & 1.75 \\
\hline Cowl maximum height, in $\left(\mathrm{H}_{\max }\right)$ & 5.36 \\
\hline Cowl aftbody length, in $\left(\mathrm{X}_{\mathrm{aft}}\right)$ & 35.82 \\
\hline Cowl aftbody boattail angle, deg & 11 \\
\hline Design Throat Mach Number & 0.70 \\
\hline
\end{tabular}

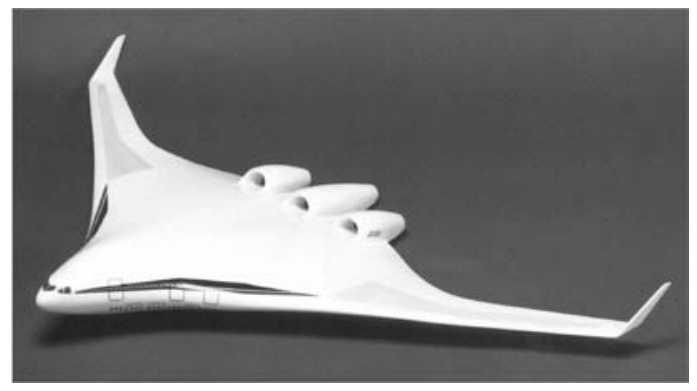

Figure 1. Blended Wing Body configuration.
Table 3. Measurement Uncertainty

\begin{tabular}{|l|l|}
\hline & \\
\hline Temperature, deg F & \pm 0.1 \\
\hline Density, slug/ $\mathrm{ft}^{3}$ & \pm 0.00001 \\
\hline Total pressure, $\mathrm{psi}$ & \pm 0.01 \\
\hline Dynamic pressure, $\mathrm{psi}$ & \pm 0.01 \\
\hline Tunnel velocity, ft/sec & \pm 1.3 \\
\hline $\mathrm{C}_{\mathrm{P}}$ & \pm 0.001 \\
\hline Actuator Mass Flow & \pm 0.35 \\
\hline Inlet Mass Flow & \pm 20 \\
\hline
\end{tabular}

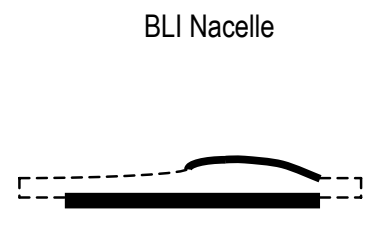

Baseline

Pylon Mounted Nacelle

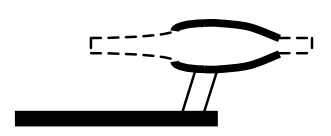

Figure 2. Control volumes for baseline and BLI nacelle. 


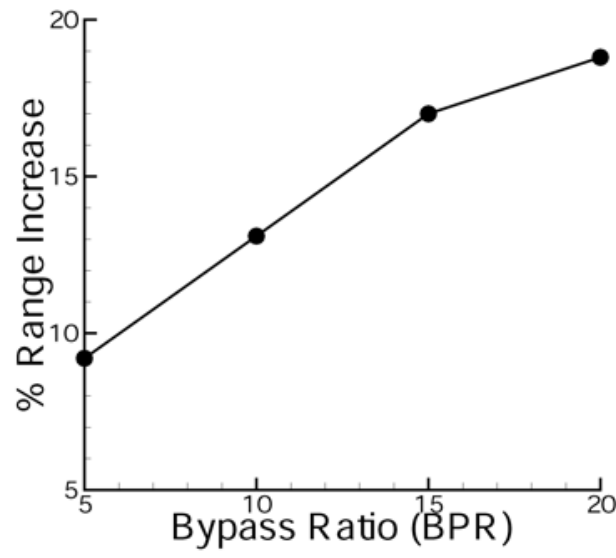

Figure 3. Effect of BLI on range increase as a function of Bypass Ratio (BPR).

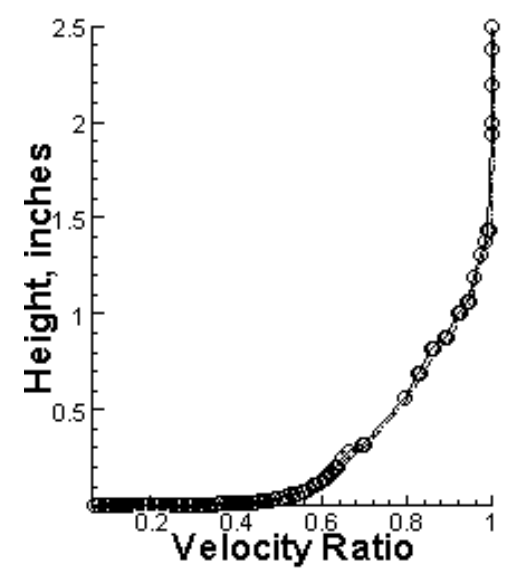

Figure 4. Boundary layer profile upstream of center of inlet, freestream Mach $=0.15$.

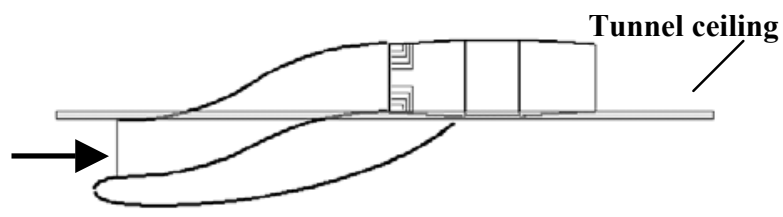

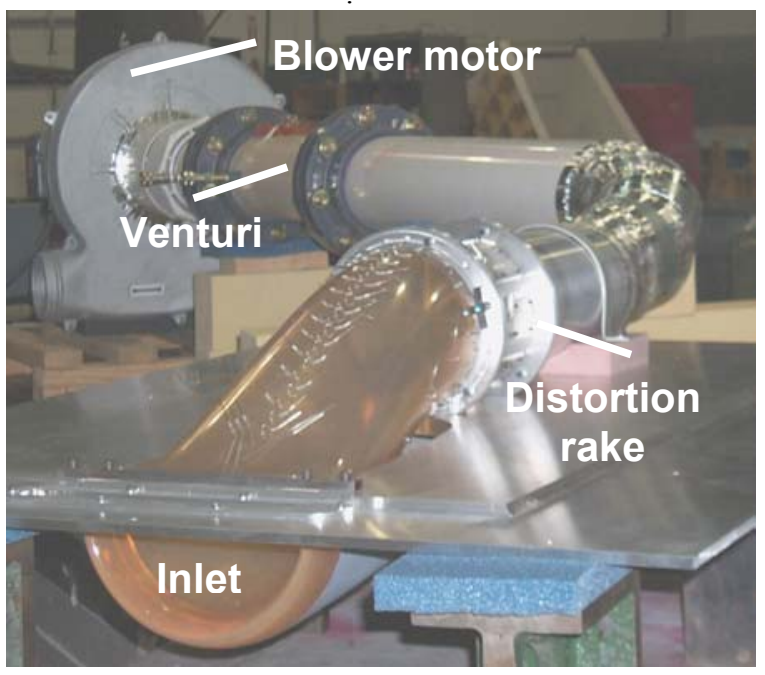

Figure $5 b$. Features of inlet model.

Figure 5. Inlet model.

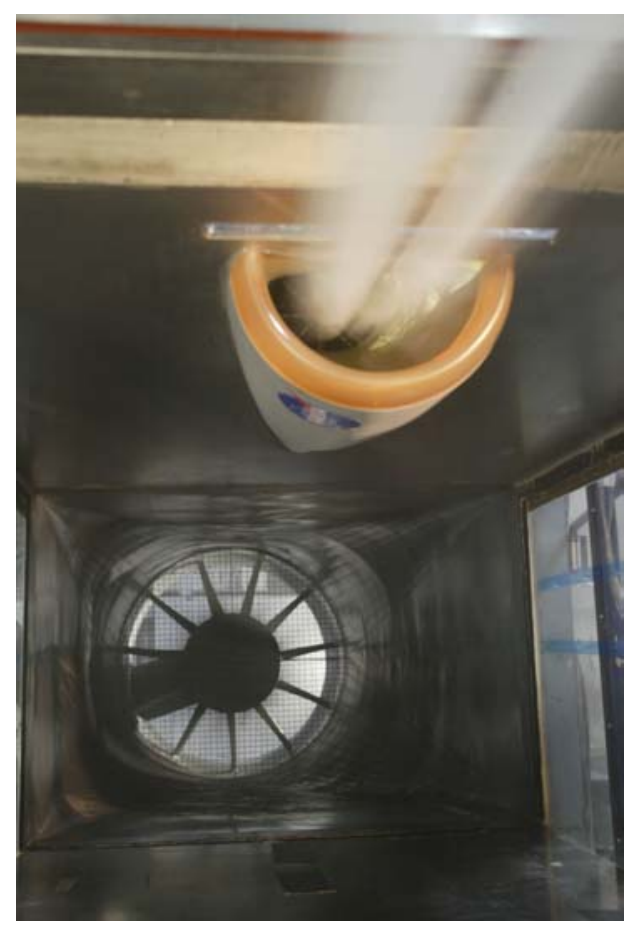

Figure 6a. Front view of inlet. BART tunnel fan also visible in background.

Figure 5a. Schematic of inlet mounted on tunnel ceiling. 


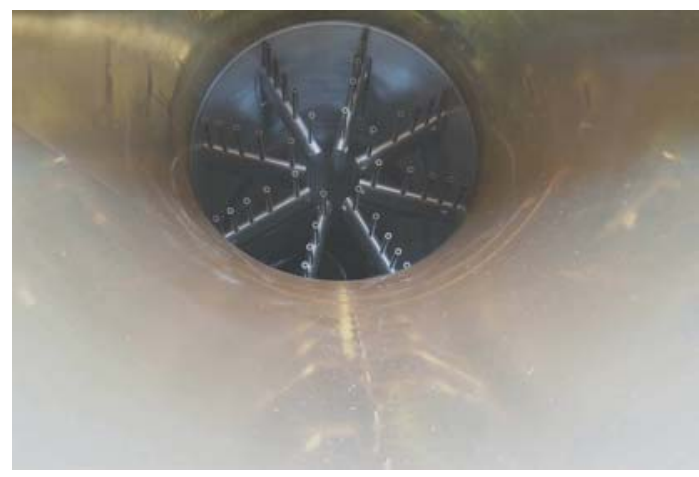

Figure $6 b$. Distortion rake installed at AIP.

Figure 6. Photographs of inlet model.

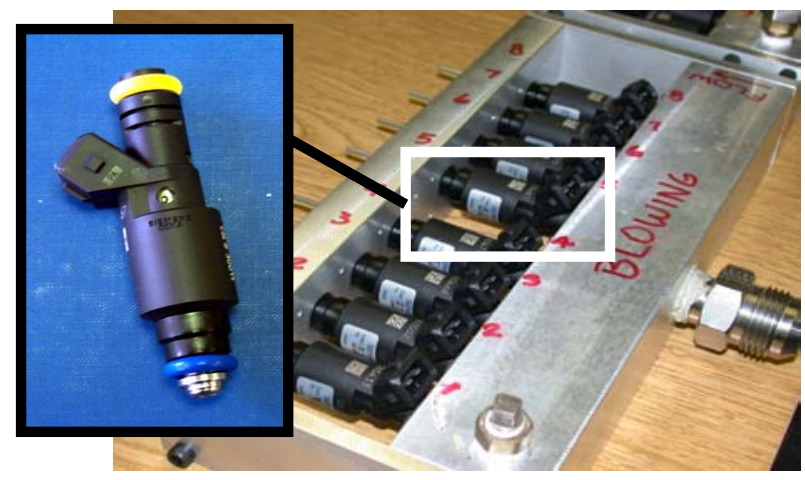

Figure 8. Actuators and manifold.
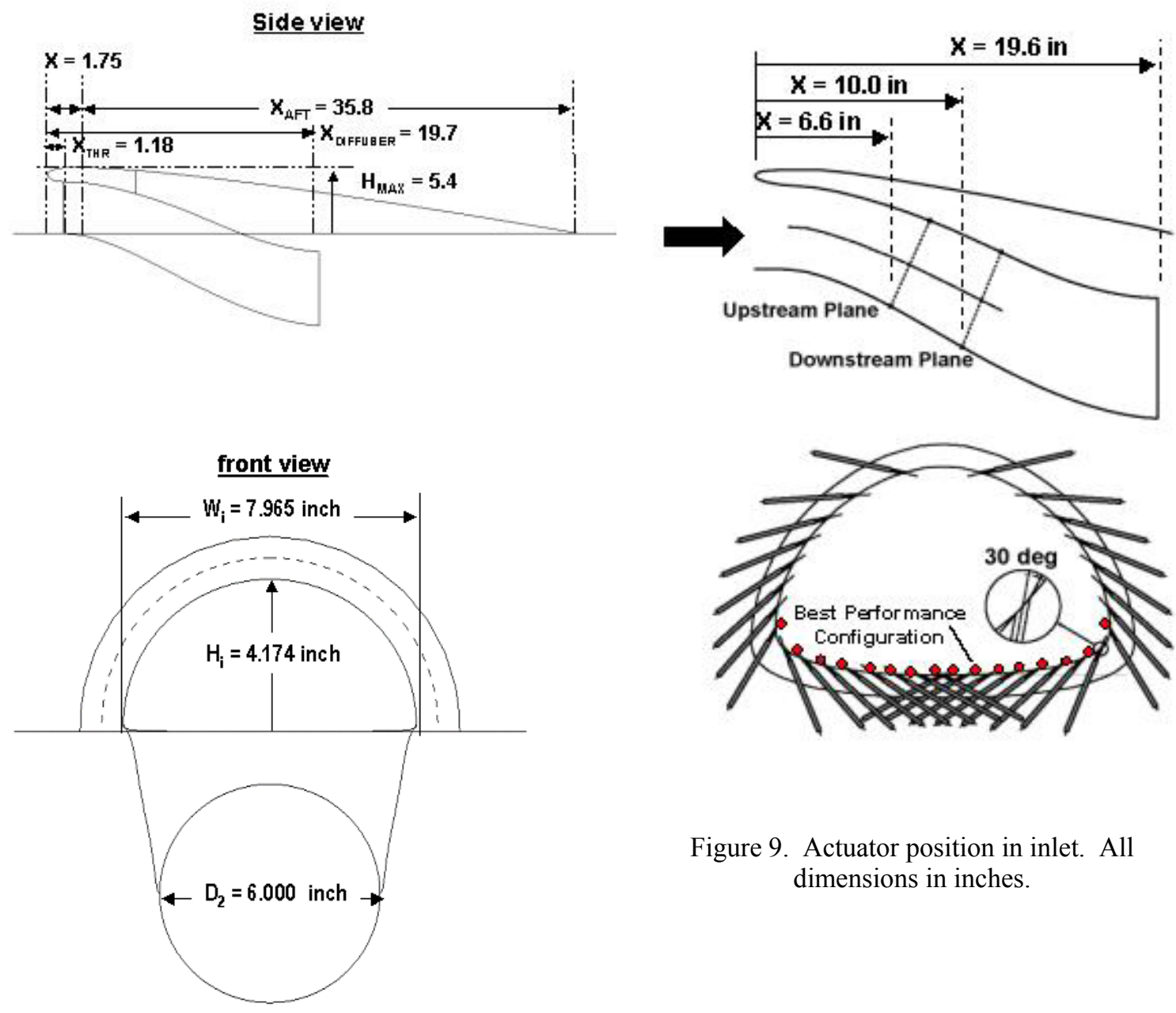

Figure 9. Actuator position in inlet. All dimensions in inches.

Figure 7. Inlet characteristics. All dimensions in inches. 


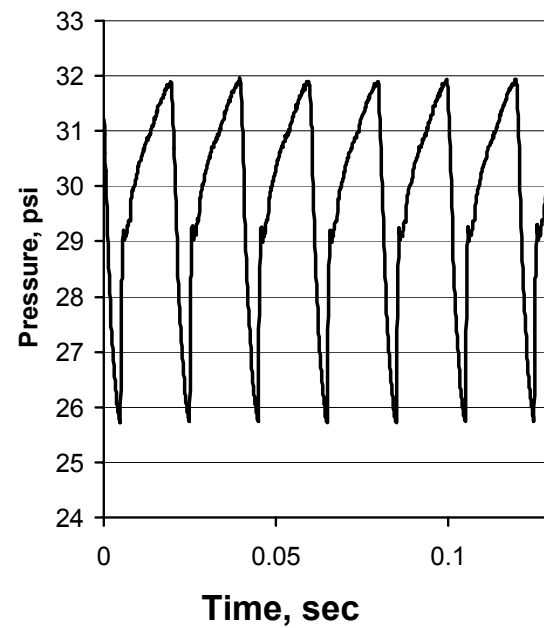

Figure 10. Actuator pressure signal.
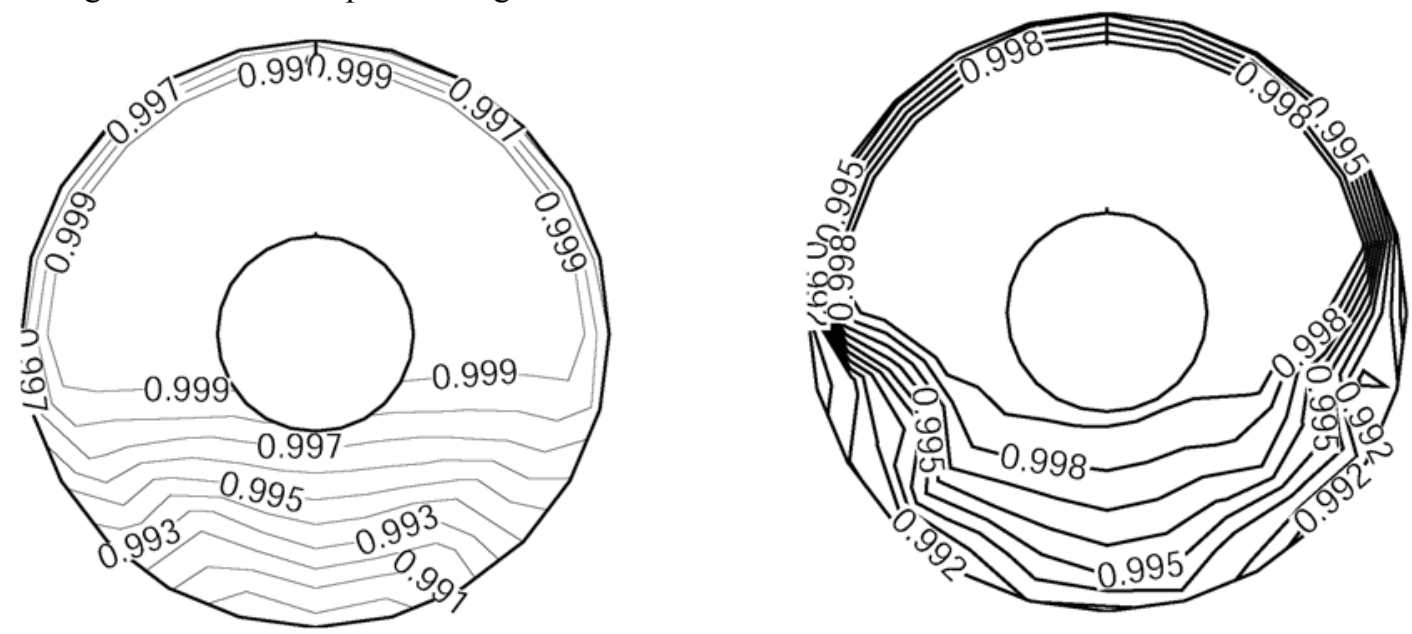

Figure 11a. Total pressure ratio contour plot from 120 rakes, $\mathrm{DC} 60=29 \%$.

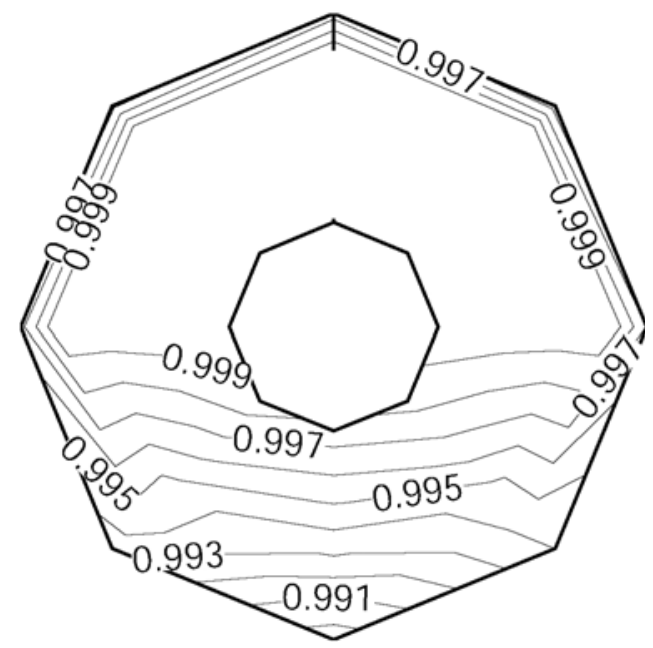

Figure $11 \mathrm{~b}$. Total pressure ratio contour plot from 40 rakes, ARP1420. DPCP $=0.0025$.

Figure 11. Total pressure ratio distribution for baseline inlet at $\mathrm{M}=0.15,1800$ SCFM.

Figure 12a. Total pressure ratio contour plot from 120 rakes, $\mathrm{DC} 60=10.8 \%$.

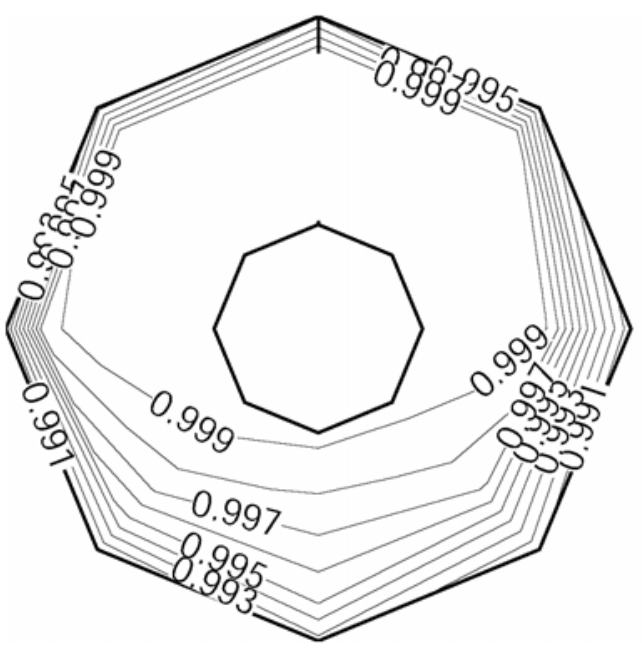

Figure 12b. Total pressure ratio contour plot from 40 rakes, ARP1420. DPCP $=0.0014$.

Figure 12. Total pressure ratio distribution for inlet with MVGs at $\mathrm{M}=0.15,1800$ SCFM. 


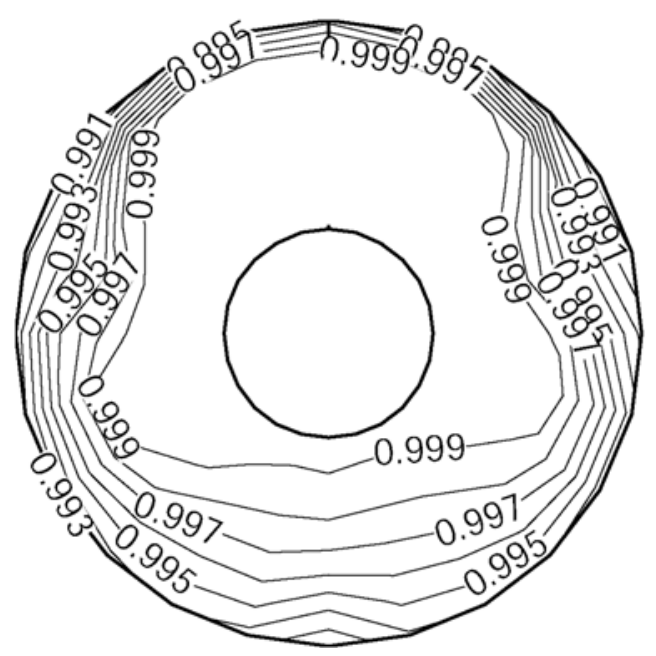

Figure 13a. Total pressure ratio contour plot from 120 rakes, DC60 $=4.6 \%$.

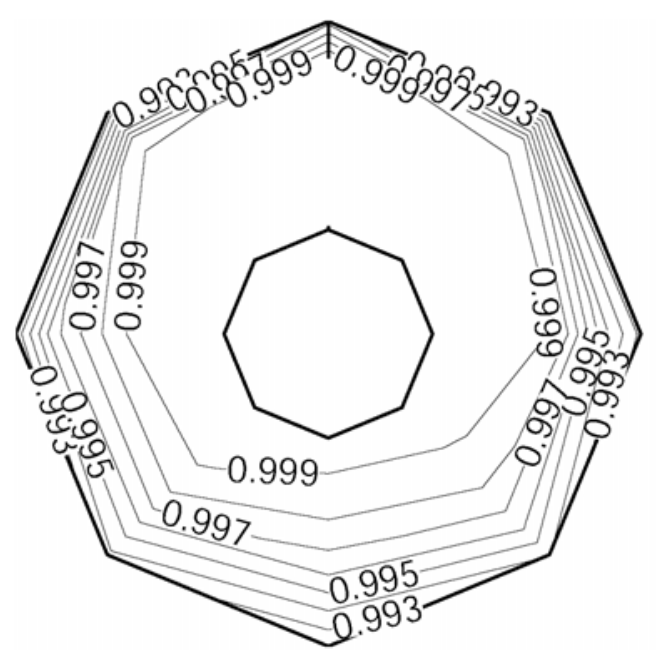

Figure 13b. Total pressure ratio contour plot from 40 rakes, ARP1420. DPCP $=0.000$.

Figure 13. Total pressure ratio distribution for inlet with $\mathrm{AFC}$ at $\mathrm{M}=0.15,1800 \mathrm{SCFM}$.

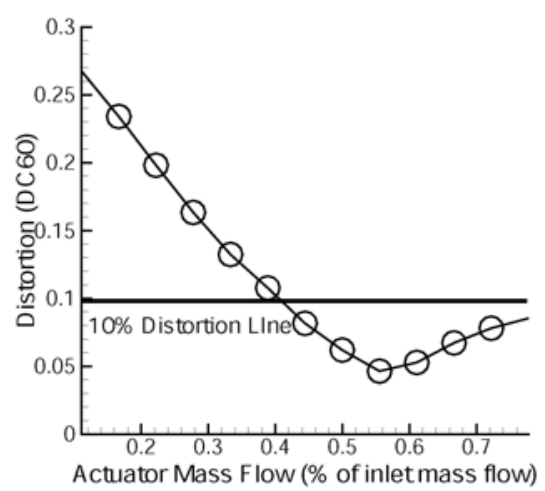

Figure 14. Actuator mass flow sweep.

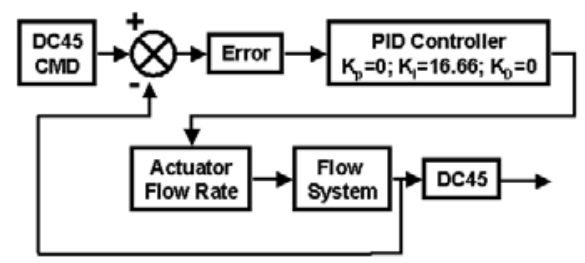

Figure 15. Diagram of control system logic.

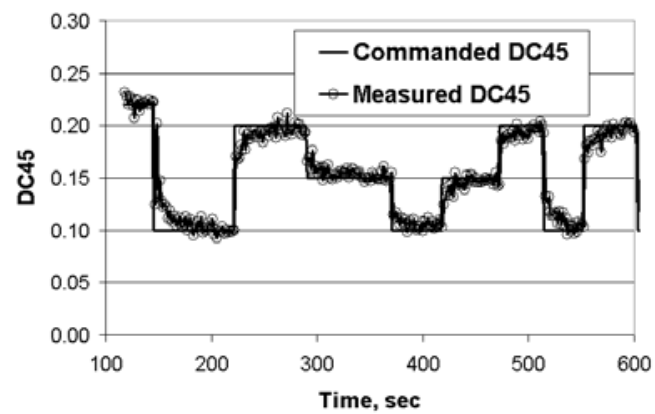

Figure 16a. Feedback variable on-line DC45.

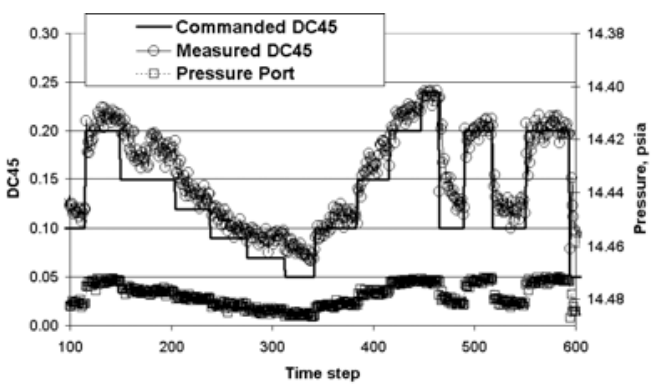

Figure 16b. Feedback variable on-line surface static pressure.

Figure 16. Effect of control system input on distortion. 